\title{
EVOLUÇÃO NOS ATRIBUTOS QUÍMICOS DE UM LATOSSOLO VERMELHO SOB DIFERENTES MÉTODOS DE PREPARO DE SOLO'
}

\section{CHEMICAL ATTRIBUTES EVOLUTION OF AN OXISOIL UNDER DIFFERENT TILLAGE METHODS}

\author{
Volnei PAULETTI ${ }^{2}$ \\ Marcelo Ricardo de LIMA ${ }^{3}$ \\ Cristina BARCIK ${ }^{4}$ \\ Andreia BITTENCOURT ${ }^{4}$
}

\begin{abstract}
RESUMO
O presente trabalho avaliou alguns atributos químicos do solo, comparando diferentes métodos de preparo de solo, após 6 anos de cultivo. Foi instalado um experimento, no município de Ponta Grossa - PR, em um LATOSSOLO VERMELHO Distrófico típico, com relevo suave ondulado, com os tratamentos: PD (plantio direto) PC (preparo convencional com uma aração e duas gradagens leves), PM (preparo mínimo com uma gradagem média e uma gradagem leve) e PDE (plantio direto com uma escarificação a cada 3 anos). O delineamento utilizado foi em blocos ao acaso com 3 repetições. A fertilização da área foi realizada com base na análise de solo. O solo foi amostrado nos anos de 1994 e 2000 em três profundidades (0-10, 10-20 e 20-30 cm), e determinaram-se os teores de $\mathrm{Ca}, \mathrm{Mg}$, e K trocáveis, $\mathrm{P}$ disponível, $\mathrm{C}$ orgânico, $\mathrm{pH}, \mathrm{H}+\mathrm{Al}$ e V. Não foram observadas diferenças no pH, $\mathrm{H}+\mathrm{Al}, \mathrm{K}$ trocável, no ano de 2000. Em algumas profundidades e tratamentos, os valores de Mg, K e P aumentaram de 1994 para 2000.
\end{abstract}

Palavras-chave: fertilidade do solo, manejo do solo, plantio direto.

\begin{abstract}
The study evaluated some soil chemical parameters related to the effects of tillage methods in the latter six years. This study was carried out in an OXISOIL, in Ponta Grossa-PR, Brazil. The treatments were: no tillage, conventionally tilled (one plow and two light grates), minimum tillage (two light grates), and soil scarification every three years (during winter). It was used the randomized blocks desing with three replications. The fertilizers were applied, based on soil analysis. Soil samples were taken in 1994 and 2000, in three layers (0-10,10-20, 20-30 cm), and evaluated for $\mathrm{Ca}, \mathrm{Mg}, \mathrm{K}, \mathrm{P}$, organic $\mathrm{C}, \mathrm{pH}, \mathrm{H}+\mathrm{Al}$. The tillage methods did not show significant effect on $\mathrm{pH}, \mathrm{H}+\mathrm{Al}, \mathrm{K}$ and $\mathrm{V}$, among methods in 2000 . In the major part of depths and treatments, $\mathrm{Mg}, \mathrm{K}$ and $\mathrm{P}$ increase from 1994 to 2000.
\end{abstract}

Key-words: soil fertility, soil tillage, no tillage.

\footnotetext{
${ }^{1}$ Trabalho apresentado na XXV Reunião Brasileira de Fertilidade do Solo e Nutrição de Plantas, de 22 a 26 de outubro de 2000 , Santa Maria - RS. ${ }^{2}$ Engenheiro Agrônomo, Mestre, Pesquisador da Fundação ABC - Castro (PR).

${ }^{3}$ Engenheiro Agrônomo (CREA 13446V-PR), Doutorando em Agronomia (PGAPV-UFPR), Professor do Departamento de Solos e Engenharia Agrícola da UFPR, Rua dos Funcionários, 1540, 80035-050, Curitiba - PR, mrlima@agrarias.ufpr.br. Autor para correspondência.

${ }^{4}$ Engenheira Agrônoma, Mestre, Bolsista RHAE/CNPQ.
} 


\section{INTRODUÇÃO}

O plantio direto, tem sido adotado em larga escala pelos produtores rurais pois é muito eficaz no controle das perdas de solo por erosão (5). No entanto, a ausência ou reduzido revolvimento do solo pode ocasionar acúmulo superficial de nutrientes (11), causado também pela deposição de resíduos na superfície, bem como o modo de aplicação de adubos no solo e o menor grau de mistura com o mesmo. A menor mobilização do solo favorece o acúmulo de Ca e Mg (4; 6), K, P e C orgânico (2), na camada de $0-5 \mathrm{~cm}$.

Os sistemas de preparo do solo com mobilização mais intensa proporcionam uma distribuição mais uniforme de nutrientes, principalmente na camada arável (4).

No entanto, as alterações na concentração e distribuição de nutrientes, $\mathrm{pH}$ e Al no perfil do solo, em função dos métodos de preparo, não deve ser limitante ao rendimento das culturas (3).

Este trabalho objetivou avaliar possíveis modificações, em diferentes profundidades, de atributos químicos de um solo conduzido sob diferentes métodos de preparo, ao longo de um período de seis anos de cultivo, em diferentes profundidades.

\section{MATERIAL E MÉTODOS}

O experimento está instalado desde 1989, no Centro de Desenvolvimento Experimental da Fundação ABC, em Ponta Grossa - PR, em um LATOSSOLO VERMELHO Distrófico típico, relevo suave ondulado, em clima do tipo Cfb segundo a classificação de Köppen.
Os métodos de preparo de solo estudados foram o PC (preparo convencional com uma aração e duas gradagens leves), PM (preparo mínimo com uma gradagem média e uma gradagem leve), PD (plantio direto com semeadura sem preparo de solo), e PDE (plantio direto com uma escarificação a cada 3 anos, realizada no inverno dos anos 1993, 1996 e 1999). O delineamento experimental utilizado foi de blocos ao acaso, com 3 repetições, e parcelas de $8,4 \times 24,0 \mathrm{~m}$. Foi empregada uma sucessão cultural usualmente utilizada na região dos Campos Gerais (Tabela 1). A fertilização da área (Tabela 1) foi realizada nas culturas de milho, soja e trigo, com base nos parâmetros estabelecidos em Raij et al. (16).

O solo foi amostrado logo após a colheita do milho das safras 1993/1994 e 1999/2000, em três profundidades (0-10, 10-20 e 20-30 cm), utilizando vinte subamostras por parcela. Nos anos de 1994 e 2000 foram determinados o $\mathrm{pH}$ em $\mathrm{CaCl}_{2}$ $0,01 \mathrm{~mol}^{\mathrm{dm}}{ }^{-3}, \mathrm{H}+\mathrm{Al}$ (obtido através de correlação com o índice SMP), Ca e Mg trocáveis (extraídos por $\mathrm{KCl} 1 \mathrm{~mol} . \mathrm{dm}^{-3}$ e determinados por complexometria com EDTA), K trocável e $P$ disponível (extraídos por $\mathrm{H}_{2} \mathrm{SO}_{4} 0,0125$ mol.dm ${ }^{-3}+$ $\mathrm{HCl} 0,05$ mol.dm ${ }^{-3}$, e determinados por fotometria de chama e colorimetria, respectivamente) (15). Com base nestes dados foi calculada a saturação por bases (V). Também foi determinado o $C$ orgânico por colorimetria (20) no ano de 2000.

$\mathrm{Na}$ avaliação estatítisca dos dados utilizouse a análise de grupos de experimentos (1), aplicando-se o teste de comparação de médias de Tukey ao nível de $5 \%$ de probabilidade.

TABELA 1 - Sucessão cultural e fertilização química utilizadas na área experimental durante o período de 1993 a 2000, em Ponta Grossa (PR).

\begin{tabular}{|c|c|c|c|c|}
\hline Safra & Cultura de inverno & $\begin{array}{c}\text { Fertilização } \\
\mathrm{N}-\mathrm{P}_{2} \mathrm{O}_{5}-\mathrm{K}_{2} \mathrm{O} \\
\left(\mathrm{kg} \cdot \mathrm{ha}^{-1}\right)\end{array}$ & Cultura de verão & $\begin{array}{c}\text { Fertilização } \\
\mathrm{N}-\mathrm{P}_{2} \mathrm{O}_{5}-\mathrm{K}_{2} \mathrm{O} \\
\left(\mathrm{kg} \cdot \mathrm{ha}^{-1}\right)\end{array}$ \\
\hline 1993 & Ervilhaca (comum) & - & Milho (cv. P.3072)* & $30-90-48$ \\
\hline 1994 & Aveia branca (cv.UPF14) & - & Soja (cv. BR- 16) & $00-60-60$ \\
\hline 1995 & Trigo (cv. BR-23)** & $10-20-20$ & Soja (cv.BR-16) & $00-60-60$ \\
\hline 1996 & Ervilhaca (comum) & - & Milho (cv.P3072) & $36-72-48$ \\
\hline 1997 & Aveia preta (comum) & - & Soja (cv.BR-16) & $00-60-60$ \\
\hline 1998 & Trigo (cv. BR-23) & $00-40-40$ & Soja (cv.BR-16) & $00-40-40$ \\
\hline 1999 & Aveia preta (comum) & - & Milho (cv. P-30 F 33) & $30-60-60$ \\
\hline 2000 & Aveia branca (Orla 9420) & - & Soja (cv. BRS 133) & $0-40-40$ \\
\hline
\end{tabular}

*No milho aplicaram-se 80 kg.ha-1 de N em cobertura, em 1993 e 1996, e 90 kg.ha-1 de N em 1999.

${ }^{* *}$ No trigo aplicaram-se $40 \mathrm{~kg} \cdot \mathrm{ha}^{-1}$ de N em cobertura em 1995, e $60 \mathrm{~kg} \cdot \mathrm{ha}^{-1}$ de N em 1998. 


\section{RESULTADOS E DISCUSSÃO}

Em relação aos teores de $C$ orgânico, não houve diferença significativa entre os tratamentos no ano de 2000 nas profundidades de $0-10$ e $20-30 \mathrm{~cm}$ (Tabela 2 e 4). Analisando as mesmas profundidades, Silveira e Stone (19), também não encontraram diferença no teror de matéria orgânica entre diferentes sistemas de preparo do solo.

Na profundidade de $10-20 \mathrm{~cm}$ (Tabela 3), o teor de C orgânico do tratamento PC foi superior ao
PD, o que pode ser atribuído ao fato do PC incorporar a matéria orgânica no preparo. A menor concentração de $C$ orgânico no plantio direto em relação ao preparo convencional também foi observada na camada de 5-10 cm, em estudo conduzido por Maria e Carmo (10). Em função das profundidades de amostragem adotadas neste experimento, não eram esperadas diferenças significativas no teor de C orgânico entre os tratamentos, pois estas devem ocorrer predominantemente até os primeiros $5 \mathrm{~cm}$ do solo (2; 11).

TABELA 2 - Valores de $\mathrm{pH}$ em $\mathrm{CaCl}_{2}, \mathrm{H}+\mathrm{Al}, \mathrm{Ca}, \mathrm{Mg}, \mathrm{K}, \mathrm{P}$ disponível, $\mathrm{C}$ orgânico, e V, na profundidade $0-10 \mathrm{~cm}$, em quatro métodos de preparo de solo, nos anos de 1994 e 2000, em um LATOSSOLO VERMELHO, em Ponta Grossa (PR).

\begin{tabular}{|c|c|c|c|c|c|c|c|c|c|c|c|c|c|}
\hline \multicolumn{2}{|c|}{$\begin{array}{l}\mathrm{pH} \mathrm{em} \\
\mathrm{CaCl}_{2}\end{array}$} & \multicolumn{2}{|c|}{$\mathrm{H}+\mathrm{Al}$} & \multicolumn{2}{|r|}{$\mathrm{Ca}$} & \multicolumn{2}{|c|}{$\mathrm{Mg}$} & \multicolumn{2}{|c|}{$\mathrm{K}$} & \multicolumn{2}{|c|}{$\begin{array}{c}\text { P disponível } \\
\left(\mathrm{mg}^{\left.-\mathrm{dm}^{-3}\right)}\right.\end{array}$} & \multirow{2}{*}{$\begin{array}{c}\text { C } \\
\text { orgânico } \\
\left(\mathrm{g} \cdot \mathrm{dm}^{-3}\right)\end{array}$} & $\begin{array}{l}\mathrm{V} \\
(\%)\end{array}$ \\
\hline$\overline{94}$ & 00 & 94 & 00 & 94 & 00 & 94 & 00 & 94 & 00 & 94 & 00 & & $\begin{array}{ll}94 & 00\end{array}$ \\
\hline 6,0 & 5,9 & $2,5 a$ & $3,9 b$ & 4,4 & $4,6 \mathrm{~B}$ & $2,3 b$ & $3,2 \mathrm{ABa}$ & $0,34 b$ & $0,58 a$ & 5,2 & $6,0 \mathrm{~B}$ & 21 & $\begin{array}{ll}72 & 68\end{array}$ \\
\hline 6,1 & 6,0 & $2,3 a$ & $3,6 b$ & 5,0 & $5,5 \mathrm{~A}$ & $2,7 b$ & $4,0 \mathrm{Aa}$ & $0,42 b$ & $0,71 a$ & 10,8 & $12.6 \mathrm{AB}$ & 22 & 77 \\
\hline 6,1 & 6,0 & $2,5 a$ & $3,9 b$ & $4,7 b$ & 5,2Aba & 2,4 & $2,8 \mathrm{~B}$ & $0,40 \mathrm{~b}$ & $0,61 a$ & 5,9 & $9,3 \mathrm{AB}$ & 22 & $75 a \quad 69 b$ \\
\hline 6,1 & 6,0 & $2,4 a$ & $3,8 b$ & $4,6 b$ & $5,1 \mathrm{Aba}$ & 2,4 & $3,1 \mathrm{AB}$ & $0,40 b$ & $0,68 a$ & $7,0 \mathrm{~b}$ & $14,0 \mathrm{Aa}$ & 25 & 69 \\
\hline \multicolumn{2}{|c|}{0,42} & \multicolumn{2}{|c|}{0,95} & \multicolumn{2}{|r|}{0,65} & \multicolumn{2}{|c|}{0,99} & \multicolumn{2}{|c|}{0,26} & \multicolumn{2}{|c|}{7,56} & 4,43 & 8,0 \\
\hline \multicolumn{2}{|c|}{0,31} & \multicolumn{2}{|c|}{0,69} & \multicolumn{2}{|r|}{0,48} & \multicolumn{2}{|c|}{0,72} & \multicolumn{2}{|c|}{0,19} & \multicolumn{2}{|c|}{5,54} & 3,25 & 5,87 \\
\hline \multicolumn{2}{|c|}{2,89} & \multicolumn{2}{|c|}{12,5} & \multicolumn{2}{|r|}{5,52} & \multicolumn{2}{|c|}{14,1} & \multicolumn{2}{|c|}{20,6} & \multicolumn{2}{|c|}{35,1} & 7,0 & 4,53 \\
\hline
\end{tabular}

Médias seguidas de letras distintas (maiúscula na coluna e minúscula na linha) diferem estatisticamente pelo teste de Tukey em nível de 5\%. DMS = diferença mínima significativa pelo teste de Tukey a 5\%. CV = coeficiente de variação.

TABELA 3 - Valores de $\mathrm{pH}$ em $\mathrm{CaCl}_{2}, \mathrm{H}+\mathrm{Al}, \mathrm{Ca}, \mathrm{Mg}, \mathrm{K}, \mathrm{P}$ disponível, $\mathrm{C}$ orgânico, e V, na profundidade $10-20 \mathrm{~cm}$, em quatro métodos de preparo de solo, nos anos de 1994 e 2000, em um LATOSSOLO VERMELHO, em Ponta Grossa (PR).

\begin{tabular}{|c|c|c|c|c|c|c|c|c|c|c|c|c|c|c|c|}
\hline \multirow[t]{2}{*}{$\begin{array}{l}\text { Trata- } \\
\text { mentos }\end{array}$} & \multicolumn{2}{|c|}{$\begin{array}{l}\mathrm{pH} \mathrm{em} \\
\mathrm{CaCl}_{2}\end{array}$} & \multirow{2}{*}{\multicolumn{2}{|c|}{$\begin{array}{ll}\mathrm{H}+\mathrm{Al} \\
94 & 00\end{array}$}} & \multicolumn{2}{|c|}{$\mathrm{Ca}$} & \multicolumn{2}{|c|}{$\mathrm{Mg}$} & \multicolumn{2}{|c|}{$\mathrm{K}$} & \multicolumn{2}{|c|}{$\begin{array}{l}\text { P disponível } \\
\left(\mathrm{mg} \cdot \mathrm{dm}^{-3}\right)\end{array}$} & \multirow{2}{*}{$\begin{array}{c}\text { C orgânico } \\
\left(\mathrm{g} \cdot \mathrm{dm}^{-3}\right)\end{array}$} & \multicolumn{2}{|c|}{$\begin{array}{c}\mathrm{V} \\
(\%)\end{array}$} \\
\hline & 94 & 00 & & & 94 & 00 & 94 & 00 & 94 & 00 & 94 & 00 & & 94 & 00 \\
\hline PC & 6,0 & 6,0 & 2,5 & 3,9 & 4,5 & 4,8 & $2,4 b$ & $3,2 a$ & $0,16 \mathrm{~b}$ & $0,34 a$ & 2,6 & $4,6 \mathrm{~B}$ & $22 \mathrm{~A}$ & 73 & 69 \\
\hline PD & 5,6 & 6,0 & 2,9 & 3,8 & 3,8 & 4,4 & $2,1 b$ & $3,2 a$ & $0,20 \mathrm{~b}$ & $0,38 a$ & $3,5 b$ & $9,0 \mathrm{Aa}$ & $18 \mathrm{~B}$ & 67 & 46 \\
\hline PM & 5,6 & 5,0 & $3,6 b$ & $5,1 a$ & 3,8 & 4,6 & $2,1 b$ & $2,8 a$ & $0,17 b$ & $0,29 a$ & $2,5 b$ & $5,0 \mathrm{Ba}$ & $19 A B$ & 62 & 67 \\
\hline PDE & 6,0 & 6,0 & 2,5 & 3,7 & 4,2 & 4,5 & $2,4 b$ & $3,2 \mathrm{a}$ & $0,19 b$ & $0,31 a$ & $1,7 b$ & $5,6 \mathrm{Ba}$ & $19 A B$ & 73 & 68 \\
\hline $\begin{array}{c}\text { DMS } \\
\text { colunas }\end{array}$ & 1 , & 13 & 2 & 13 & & 20 & 0, & & & & & 72 & 3,88 & 33 & 7 \\
\hline $\begin{array}{l}\text { DMS } \\
\text { linhas }\end{array}$ & 0 & 83 & 1, & 56 & & 38 & 0 & & & & & 99 & 2,85 & &, 7 \\
\hline CV (\%) & 8 & 0 & 2 & 4 & & ,4 & 13 & & & & & 5,7 & 7,15 & 21 &, 1 \\
\hline
\end{tabular}

Médias seguidas de letras distintas (maiúscula na coluna e minúscula na linha) diferem estatisticamente pelo teste de Tukey em nível de 5\%. DMS = diferença mínima significativa pelo teste de Tukey a 5\%. CV = coeficiente de variação. 
Não foram observadas diferenças significativas de $\mathrm{H}+\mathrm{Al}$ e $\mathrm{V}$ entre os métodos de preparo de solo estudados, nas três profundidades (Tabelas 2, 3 e 4). Com relação ao pH, somente na camada de $20-30 \mathrm{~cm}$, observou-se que, no sistema PDE, o $\mathrm{pH}$ foi significativamente maior que no PM em 1994 (Tabela 3). No entanto, não se observou nenhuma diferença significativa de $\mathrm{pH}$ entre os tratamentos em 2000, de forma semelhante ao observado por outros autores $(2 ; 3 ; 7 ; 17)$. O pH, a acidez potencial $(\mathrm{H}+\mathrm{Al})$ e a saturação de bases $(\mathrm{V})$ estão intrinsecamente ligados, e como não houve calagem na área, era esperada a ausência de respostas dos métodos de preparo nestas três variáveis no ano de 2000. Ao se comparar os anos, constata-se que, em todos os tratamentos, o $\mathrm{H}+\mathrm{Al}$ $(0-10 \mathrm{~cm})$ é maior em 2000 do que em 1994, o que não se refletiu no $\mathrm{pH}$ do solo (Tabela 2). Em todos os tratamentos aumentou o pH de 1994 para 2000, na profundidade de $20-30 \mathrm{~cm}$ (Tabela 4).

TABELA 4 - Valores de $\mathrm{pH}$ em $\mathrm{CaCl}_{2}, \mathrm{H}+\mathrm{Al}, \mathrm{Ca}, \mathrm{Mg}, \mathrm{K}, \mathrm{P}$ disponível, $\mathrm{C}$ orgânico, e V, na profundidade $20-30 \mathrm{~cm}$, em quatro métodos de preparo de solo, nos anos de 1994 e 2000, em um LATOSSOLO VERMELHO, em Ponta Grossa (PR).

\begin{tabular}{|c|c|c|c|c|c|c|c|c|c|c|c|c|c|c|}
\hline \multirow[t]{2}{*}{$\begin{array}{l}\text { Trata- } \\
\text { mentos }\end{array}$} & \multicolumn{2}{|c|}{$\mathrm{pH} \mathrm{em} \mathrm{CaCl}_{2}$} & \multicolumn{2}{|c|}{$\mathrm{H}+\mathrm{Al}$} & \multicolumn{2}{|l|}{$\mathrm{Ca}$} & \multicolumn{2}{|c|}{$\mathrm{Mg}$} & \multicolumn{2}{|c|}{$\mathrm{K}$} & \multicolumn{2}{|c|}{$\begin{array}{l}\text { P disponível } \\
\left(\mathrm{mg} \cdot \mathrm{dm}^{-3}\right)\end{array}$} & \multirow{2}{*}{$\begin{array}{c}\begin{array}{c}\mathrm{C} \\
\text { Orgânico } \\
\left(\mathrm{g} \cdot \mathrm{dm}^{-3}\right)\end{array} \\
00\end{array}$} & $\begin{array}{l}\mathrm{V} \\
(\%)\end{array}$ \\
\hline & 94 & 00 & 94 & 00 & 94 & 00 & 94 & 00 & 94 & 00 & 94 & 00 & & $94 \quad 00$ \\
\hline $\mathrm{PC}$ & $5,4 \mathrm{ABb}$ & $5,9 a$ & 3,6 & 3,9 & $2,5 \mathrm{ABb}$ & $3,6 a$ & $1,7 b$ & $2,9 a$ & $0,14 b$ & $0,23 a$ & $0,6 b$ & $1,6 a$ & 16 & $54 b \quad 66 a$ \\
\hline PD & $5,3 \mathrm{ABb}$ & $6,0 a$ & 3,6 & 4,0 & $2,2 \mathrm{Bb}$ & $3,5 \mathrm{a}$ & $1,5 b$ & $2,9 a$ & $0,17 b$ & $0,26 a$ & $0,7 b$ & $2,3 a$ & 16 & $51 \quad 61$ \\
\hline PM & $5,1 \mathrm{Bb}$ & $6,0 a$ & 4,5 & 3,8 & $2,4 \mathrm{ABb}$ & $3,7 a$ & $1,6 b$ & $2,7 a$ & 0,12 & 0,19 & $0,6 b$ & $2,6 a$ & 16 & $48 b \quad 63 a$ \\
\hline PDE & $5,6 A b$ & $6,1 a$ & 3,0 & 3,6 & $2,8 \mathrm{Ab}$ & $3,4 a$ & $1,7 b$ & $2,9 a$ & 0,14 & 0,20 & $0,5 b$ & $2,3 a$ & 15 & 60 \\
\hline $\begin{array}{l}\text { DMS } \\
\text { colunas }\end{array}$ & 0,3 & & & 46 & $\overline{0,4 \epsilon}$ & & 0 , & & & & & & 3,19 & 13,9 \\
\hline $\begin{array}{c}\text { DMS } \\
\text { linhas }\end{array}$ & 0,2 & & & & 0,33 & & 0 & & & & & & 2,34 & 10,24 \\
\hline$\overline{C V}(\%)$ & 2,7 & & & & 6,25 & & 13 & 4 & & & & & 7,2 & 9,8 \\
\hline
\end{tabular}

Médias seguidas de letras distintas (maiúscula na coluna e minúscula na linha) diferem estatisticamente pelo teste de Tukey em nível de 5\%. DMS = diferença mínima significativa pelo teste de Tukey a 5\%.CV = coeficiente de variação.

Não houve diferença significativa nos teores de $\mathrm{K}$ trocável, entre os métodos de preparo de solo, semelhante ao encontrado por Santos et al. (17) ao comparar preparo convencional e plantio direto. Em função das altas quantidades absorvidas e posteriormente restituídas ao solo à partir da decomposição da parte aérea, o $\mathrm{K}$ trocável tende a se acumular na superfície do solo (11), como pode ser observado na profundidade de $0-10 \mathrm{~cm}$ (Tabela 2) em comparação às demais camadas (Tabelas 3 e 4). Devido às adubações efetuadas (Tabela 1), o teor de K trocável aumentou significativamente de 1994 para 2000 em todos os tratamentos, nas profundidades de 0-10 e 10-20 cm (Tabelas 2 e 3).

Em 1994 não houve diferença significativa no teor de fósforo entre os tratamentos em todas as profundidades. Mudanças no teor de fósforo, entre sistemas de manejo do solo podem demorar a ocorrer, podendo levar períodos de até 7 anos para se observarem modificações à $10 \mathrm{~cm}$ (10). Deve ser ressaltado que os coeficientes de variação (CV) encontrados para $\mathrm{P}$ e $\mathrm{K}$ foram altos em comparação com as demais variáveis, o que era esperado na medida em que estes atributos químicos apresentam elevada variabilidade amostral em plantio direto (18). O P disponível na camadas $0-10 \mathrm{~cm}$ apresentou, no ano de 2000, maior concentração no tratamento PDE em relação ao PC (Tabela 2). De forma semelhante, outros estudos tem encontrado maior concentração de $P$ na camada de $0-5 \mathrm{~cm}$ no plantio direto em relação ao convencional $(11 ; 17)$. Nas demais profundidades em todos os sistemas, tal elemento teve valores classificados como baixos (12), devido à pouca mobilidade deste nutriente. Estes resultados são concordantes com aqueles obtidos por Bayer e Bertol (2), que encontraram menor teor de P com o aumento da profundidade, em todos os sistemas de manejo de solo e rotação de culturas que estes autores estudaram. Este aspecto pode ser explicado pela maior concentração na superfície, devido menor homogeneização do $\mathrm{P}$ com o solo no plantio direto.

Na camada de $10-20 \mathrm{~cm}$ e no ano de 2000, o PD apresentou maior teor de $\mathrm{P}$ que os demais tratamentos (Tabela 3).

Os teores de $\mathrm{P}$ nas profundidades de 10-20 e 20-30 cm aumentaram de 1994 para 2000, apesar da baixa mobilidade deste nutriente. A elevação do teor de $\mathrm{P}$ no solo pode ser justificada pelo uso de adubos fosfatados, que elevam gradativamente a quantidade deste elemento no solo (8). Apesar da baixa mobilidade do $P$, em 2000 os teores deste nutriente são significativamente maiores do que em 1994 em todos os tratamentos, mesmo na profundidade $20-30$ $\mathrm{cm}$, o que pode estar relacionado a fatores como a 
decomposição da matéria orgânica (especialmente as raízes) no solo.

Os teores de $\mathrm{Ca}$ trocável foram significativamente superiores no tratamento PD em relação ao PC na camada 0-10 cm em 2000 (Tabela 2), mostrando que este elemento está se acumulando na camada superficial do solo no tratamento PD, semelhante ao observado por Merten e Mielniczuk (11) na camada $0-5 \mathrm{~cm}$. O magnésio apresentou-se significativamente superior no tratamento PD (4 $\mathrm{cmol}\left(\right.$ carga). $\mathrm{dm}^{-3}$ ) em relação ao $\mathrm{PM}$ na profundidade de 0-10 cm em 2000 (Tabela 2) enquanto que nas demais profundidades não houve efeito dos tratamentos.

Em alguns tratamentos e profundidades houve aumento dos teores de $\mathrm{Ca}$ e Mg trocáveis disponível do ano de 1994 para 2000 (Tabelas 2, 3 e 4). Valores de Ca e Mg trocáveis, superiores aos valores medidos antes do início do experimento, foram obtidos por Santos et al. (17) comparando preparo convencional e plantio direto. O aumento do $\mathrm{Ca}$ pode ser devido à presença deste nutriente em muitas formulações de NPK utilizadas neste experimento, enquanto o aumento de $\mathrm{Mg}$ pode estar associado à decomposição da matéria orgânica, favorecida pela melhoria das condições de fertilidade do solo.

No entanto, o aumento da fertilidade química de 1994 para 2000, evidenciado pelo aumento de teores de $\mathrm{Mg}, \mathrm{P}$ e $\mathrm{K}$ em muitas profundidades e tratamentos, não se refletiu em maiores rendimentos de grãos de soja (9), milho (13) e trigo (14), nesta mesma área experimental, sugerindo que poderiam ser revistas as recomendações de adubação atuais.

\section{CONCLUSÕES}

Não foram observadas diferenças dos parâmetros $\mathrm{pH}, \mathrm{H}+\mathrm{Al}, \mathrm{K}$ trocável e $\mathrm{V}$ entre os métodos de preparo de solo, no ano de 2000 . Os valores de $\mathrm{Ca}$ e $\mathrm{Mg}$ aumentaram em 2000 na profundidade de 0-10 $\mathrm{cm}$ no tratamento $\mathrm{PD}$ em relação ao $\mathrm{PC}$ e $\mathrm{PM}$, respectivamente. O $\mathrm{P}$ foi maior no $\mathrm{PDE}$ na camada de $0-10 \mathrm{~cm}$ em relação ao $\mathrm{PC}$, e no $\mathrm{PD}$ na camada de 10 $20 \mathrm{~cm}$ em relação aos demais tratamentos, no ano de 2000. Em algumas profundidades e tratamentos os teores de $\mathrm{Ca}, \mathrm{Mg}, \mathrm{K}$ e $\mathrm{P}$ aumentaram de 1994 para 2000.

\section{AGRADECIMENTO}

Ao Conselho Nacional de Desenvolvimento Científico e Tecnológico (CNPq) pelo apoio através da concessão de bolsas do programa RHAE aos pesquisadores deste projeto.

\section{REFERÊNCIAS}

1. BANZATTO, D.A.; KRONKA, S.N. Experimentação agrícola. 2.ed. Jaboticabal: FUNEP, 1992. Cap. 8: Análise de grupos de experimentos. p.213-236.

2. BAYER, C.; BERTOL, I. Características químicas de um Cambissolo Húmico afetadas por sistemas de preparo, com ênfase na matéria orgânica. Revista Brasileira de Ciência do Solo, Viçosa, v.23, n.3, p.687-694, 1999.

3. BAYER, C.; MIELNICZUK, J. Características químicas do solo afetadas por métodos de preparo e sistemas de cultura. Revista Brasileira de Ciência do Solo, Campinas, v.21, n.1, p.105-112, 1997.

4. ELTZ, F.L.F.; PEIXOTO, R.T.G.; JASTER, F. Efeitos de sistemas de preparo do solo nas propriedades físicas e químicas de um Latossolo Bruno álico. Revista Brasileira de Ciência do Solo, Campinas, v.13, n.2, p.259-267, 1989.

5. HERNANI, L.C.; SALTON, J.C.; FABRÍCIO, A.C.; DEDECEK, R.M.; ALVES JÚNIOR, M. Perdas por erosão e rendimento de soja e de trigo em diferentes sistemas de preparo de um Latossolo Roxo de Dourados (MS). Revista Brasileira de Ciência do Solo, Viçosa, v.21, n.4, p.667-676, 1997.

6. ISMAIL, I.; BLEVINS, R.L.; FRYE, W.W. Long-term no tillage effects on soil properties and continuous corn yields. Soil Science Society of America Journal, Madison, v.58, n.1 p.193-198, 1994.

7. KLEPKER, D.; ANGHINONI, I. Características físicas e químicas do solo afetadas por métodos de preparo e modos de adubação. Revista Brasileira de Ciência do Solo, Campinas, v.19, n.3, p.395-401, 1995.

8. LANTMANN, A.F. Manejo de fósforo e potássio para a soja em plantio direto. In: SIMPÓSIO SOBRE FERTILIDADE DO SOLO E NUTRIÇÃO DE PLANTAS NO SISTEMA DE PLANTIO DIRETO (1.: Ponta Grossa: 2000). Anais. Ponta Grossa: Associação dos Engenheiros Agrônomos dos Campos Gerais, 2000. p.137-145.

9. LIMA, M.R.; BITTENCOURTA.; BARCIK, C.;PAULETTI, V.; TESSARO, L.C. Rendimento de grãos de soja em uma sucessão cultural sob diferentes sistemas de preparo e semeadura. IN: REUNIÃO BRASILEIRA DE MANEJO E CONSERVAÇÃO DO SOLO E DA ÁGUA (13.: Ilhéus: 2000). Anais. Ilhéus: Sociedade Brasileira de Ciência do Solo, 2000. 1 CD-ROM.

10. MARIA, I.C.; CARMO, O.M. Fósforo, potássio e matéria orgânica em um Latossolo Roxo, sob sistemas de manejo com milho e soja. Revista Brasileira de Ciência do Solo, Campinas, v.17, n.3, p.471-477, 1993.

11. MERTEN, G.H.; MIELNICZUK, J. Distribuição de sistema radicular e dos nutrientes em Latossolo Roxo sob dois sistemas de preparo de solo. Revista Brasileira de Ciência do Solo, Campinas, v.15, n.3, p.369-374, 1991.

12. OLEYNIK, J.; BRAGAGNOLO, N.; BUBLITZ, U.; SILVA, J.C.C. Análises de solo: tabelas para transformação de resultados analíticos e interpretação de resultados. 3. ed. Curitiba: EMATER-Paraná, 1995. 66p. (Informação técnica 31).

13. PAULETTI, V.; BARCIK, C.; LIMA, M.R.; BITTENCOURT, A. Rendimento de grãos de milho em uma sucessão cultural sob diferentes sistemas de preparo de solo e semeadura. In: CONGRESSO BRASILEIRO DE CIÊNCIA DO SOLO (28.: Londrina: 2001). Resumos. Londrina: Sociedade Brasileira de Ciência do Solo, 2001. p.259. 
PAULETTI, V. et al. Evolução nos atributos químicos...

14. PAULETTI, V.; LIMA, M.R.; BARCIK, C.; BITTENCOURT, A.; PALLONI, C. Rendimentos de grãos de trigo em uma sucessão cultural sob diferentes sistemas de preparo de solo e semeadura. In: CONGRESSO BRASILEIRO DE CIÊNCIA DO SOLO (28.: Londrina: 2001). Resumos. Londrina: Sociedade Brasileira de Ciência do Solo, 2001. p.286.

15. PAVAN, M.A.; BLOCH, M.F.; ZEMPULSKI, H.C.; MIYAZAWA, M.; ZOCOLER, D.C. Manual de análise química de solo e controle de qualidade. Londrina: IAPAR, 1992. 40p. (Circular 76).

16. RAIJ, B. van; CANTARELLA, H.; QUAGGIO, J.A.; FURLANI (Eds.). Recomendações de adubação e calagem para o Estado de São Paulo. 2.ed. Campinas: Instituto Agronômico, Fundação IAC, 1996. 285p. (Boletim técnico 100).

17. SANTOS, H.P.; TOMM, G.O.; LHAMBY, J.C.B. Plantio direto versus convencional: efeito na fertilidade do solo e no rendimento de grãos de culturas em rotação com cevada. Revista Brasileira de Ciência do Solo, Campinas, v.19, n.3, p.449-454, 1995.

18. SCHILINDWEIN, J.A.;ANGHINONI, I. Variabilidade horizontal de atributos de fertilidade e amostragem do solo no sistema plantio direto. Revista Brasileira de Ciência do Solo, Viçosa, v.24, n.1, p.85-91, 2000.

19. SILVEIRA, P.M.; STONE, L.F. Teores de nutrientes e de matéria orgânica afetados pela rotação de culturas e sistemas de preparo de solo. Revista Brasileira de Ciência do Solo, Viçosa, v.25, n.2, p.387-394, 2001.

20. TEDESCO, M.J.; VOLKWEISS, S.J.; BOHNEN, H. Análise de solo, plantas e outros materiais. Porto Alegre: Universidade Federal do Rio Grande do Sul, Departamento de Solos, 1985. 188 p.

Recebido em 10/05/2002 Aceito em 04/10/2005 